\title{
Native advertising and the credibility of online publishers
}

\author{
Manon Revel, Amir Tohidi \\ Institute for Data, Systems and Society, Massachusetts Institute of Technology, Cambridge, MA 02139, \\ mrevel@mit.edu, atohidi@mit.edu \\ Dean Eckles \\ Sloan School of Management, Massachusetts Institute of Technology, Cambridge, MA 02139 eckles@mit.edu \\ Adam Berinsky \\ Department of Political Science, Massachusetts Institute of Technology, Cambridge, MA 02139, berinsky@mit.edu \\ Ali Jadbabaie \\ Institute for Data, Systems and Society, Laboratory for Information and Decision Systems, Civil Engineering Department, \\ Cambridge, MA 02139, jadbabai@mit.edu
}

\begin{abstract}
The digitization of news publishing has resulted in new ways for advertisers to reach readers, including native advertising formats that blend in with news. However, native ads can redirect attention off-site and affect the readers' impression of the publishers. Using a combination of observations of ad content across many publishers and two large randomized experiments, we investigate the characteristics of a pervasive native ad format and compare the impact of different native ads characteristics on perceived news credibility. Analyzing 1.4 million collected ad headlines, we found that over $80 \%$ of these ad headlines have a clickbait-style, and that politics is among the most common topics in ads. In two randomized experiments (combined $n=9,807$ ), we varied the style and content of native ads embedded in news articles and asked people to assess the articles' credibility. An initial experiment suggested that different publishers were impacted differently by the ads. We conducted a more sophisticated experiment $(n=5,040)$ - using hundreds of unique combinations of ads, articles, and publishers - to study effects of clickbait and political ads. Findings from this pre-registered experiment provide evidence that clickbait and, to a lesser extent, political ads, substantially reduce readers' perception of the articles' credibility. This phenomenon is driven by the least well-known publishers and by readers' prior familiarity with those publishers. Importantly, we rule out large effects of non-clickbait ads, compared with no ads, on readers' attitudes. Many publishers using clickbait native ads may trade short-term revenues for audience trust.
\end{abstract}

Key words: advertising, newspapers, political communication

\section{Introduction}

In 2016 two New York Times journalists drew attention to "Around the Web" ads readers see at the bottom of news articles (Maheshwari and Herrman 2016). These ads appear on the majority of 
online news publisher websites, and are "often augmented with eye-catching photos and curiositystoking headlines about the latest health tips, celebrity news or ways to escape financial stress" (Maheshwari and Herrman 2016) (for example see Figure 1). The journalists raised concerns about the quality of these ads and echoed publishers' worries regarding the potential for harm to their credibility. In this paper, we systematically examine this hypothesis.

Figure 1 Native Ads examples.
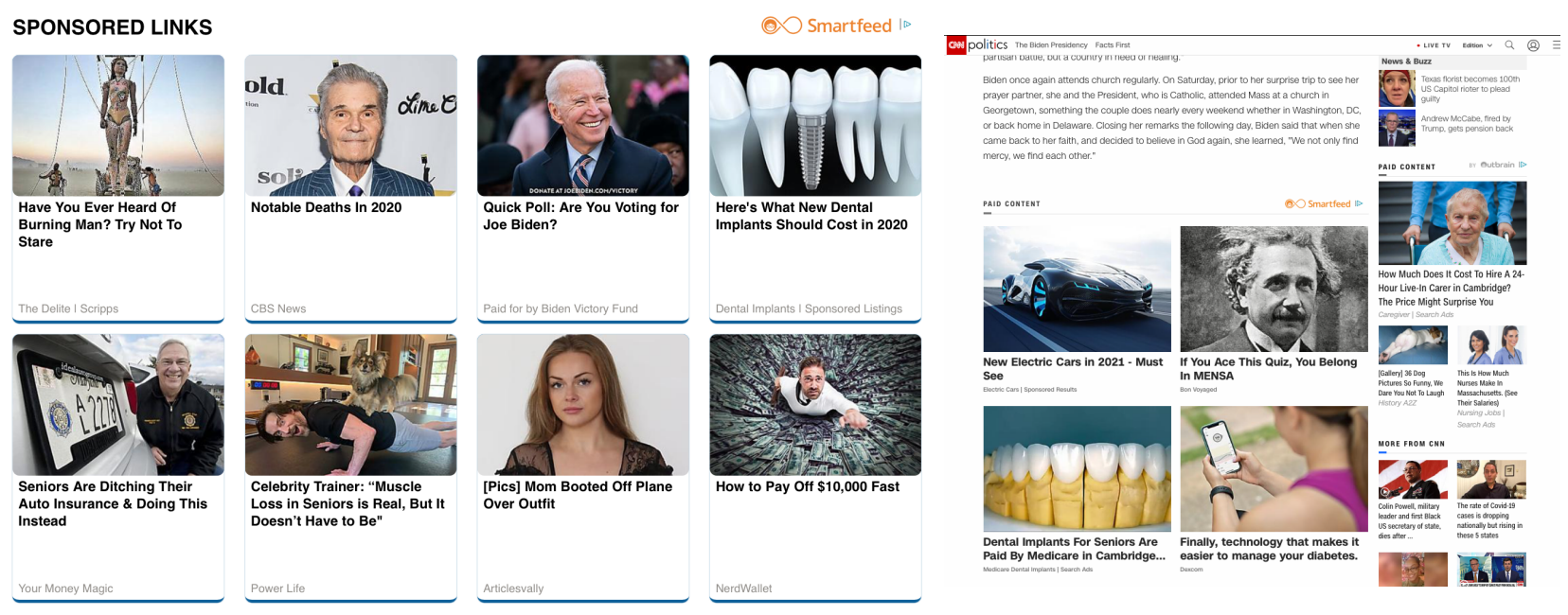

Note. Recommended content-type native ads displayed by Outbrain on The Denver Post (left) and in context on $C N N$ at the bottom and alongside an article (right). These ads appear below, alongside, or within an article and often are conjoined with non-advertising recommendations from the publisher of the article. They are typically signaled with these headers like "Around the Web Content", "Recommended for You" or "Sponsored Links."

High-credibility and trusted media can help foster meaningful mass participation in a democracy. An independent press can educate and engage the public (Benson 2019), enhance political participation (Gentzkow et al. 2011), and help citizens to hold officials accountable (Snyder and Strömberg 2010). However, media credibility seems to be decreasing (Pew Research Center 2012, Brenan 2016, Fink 2019, Usher 2018, Lewis 2020), while the media industry also faces financial difficulties. Increasingly, news organizations have turned toward online advertising to increase their revenue and provide financial stability. The written press has been particularly impacted by these trends - digital advertising makes up an increasing portion of online publishers' revenue (Pew Research Center 2019a,b).

In addition to the standard "banner" display ads, another type of advertising has been on the rise over the past decade: native ads. Native ads adopt the format, appearance, and even conventions of the non-advertising content that surrounds it. Per Microsoft's classification, ${ }^{1}$ such native ads

\footnotetext{
${ }^{1}$ See: https://about.ads.microsoft.com/en-us/blog/post/april-2021/what-is-native-advertising
} 
are sometimes placed on social media platforms and appear as regular posts, on search engines listing and look exactly like any other non-commercial result. They also appear on news publishers' website as premium (articles hosted by news publishers but written by advertisers for commercial purposes) or in-feed promoted content. This latest category (in-feed promoted content) is the object of our work. In the context of news, they appear below, alongside, or within an article and often are conjoined with non-advertising recommendations from the publisher of the article. They are typically signaled with these headers like "Around the Web Content," "Recommended for You" or "Sponsored Links." To be sure, the native ads we study do not typically blend with the body of articles, but they are instead mixed with recommended articles that are legitimate "next read" from the host publishers.

This native ad inventory (i.e., opportunities to show such ads) is aggregated by an outside company, whose role is to match a collection of advertisers, users, and news publishers. Payments by native ad networks to publishers constitute a large fraction of the publishers' overall revenues (Sirrah 2019, Perrin 2019). But such ads may come with other costs. Placing dubious ads camouflaged as articles on news sites could route traffic away from the publisher and diminish the perceived credibility of the host publishers. Thus, online news publications using such native advertising may be trading short-term financial survival with long-term perceived credibility.

The two largest native ad networks are Taboola and Outbrain. The former claims that consumers prefer the "soft-sell approach" of native ads to the "visible" approach of display ads (Hurley Hall 2019). However, the president of Slate, an online publication, warned, "It is not the right look if you're trying to say you're a high-quality, upper-tier website — if you have something like this [i.e., native ads] on it - and I think it's time for us to be honest about that" (Maheshwari and Herrman 2016).

Native ads may borrow some of the publisher's credibility if readers are more likely to click on ads camouflaged to appear like news articles rather than an obvious advertisement. Despite the Federal Trade Commission's regulations on advertisement disclosure, the native ads' potential for deception has raised concerns among journalists, researchers and legislators. More precisely, native ads have been criticized for not prominently disclosing that they are paid placements, for displaying low-quality ads, ad content that is too closely related to the news content (Griffith 2014, Marshall 2013, Rey 2013, Bashir et al. 2016), and for making it difficult for readers to recognize native ads (Bakshi 2014, Wojdynski 2019). Further, there are concerns about native ads muddling the advertising-editorial boundary (Carlson 2015, Wojdynski 2019, Iversen and Knudsen 2019), misleading readers and contributing to the diminishing credibility of journalism (Gilley 2013, Austin and Newman 2015, Schauster et al. 2016, Maheshwari and Herrman 2016), and violating the 2015 U.S. Federal Trade Commission disclosure regulations (Federal Trade Commission (FTC) 2015, 
Bashir et al. 2016). Some publishers have announced that they are removing native ads because they conflict with their priority: "When you're looking at things from that prism and you're not maniacally obsessed with monetizing every single pixel, Outbrain is very obviously not fitting into your equation anymore," [Slate's president] said. "If your readers' trust and loyalty is No. 1 as the thing you care about most, you can't have that on your page." (Maheshwari and Herrman 2016). However, several publishers have then backtracked and started running native ad formats again.

Native ads have further been under scrutiny for displaying political information. While the impact of political ads on media's credibility has been discussed before Svetlik (2016), the fact that publishers have no control over the ads they host through the native ads network can be particularly worrisome. For one, conservative-leaning ads may appear in both conservative and democratic publications, creating conflicting situations for publishers that aim to retain a targeted audience. Furthermore, ads featuring political candidates or topics are hardly comparable to ads centered around celebrities. Entertainment ads may work because of pure sensationalism, but we believe that political ads may generate distinctive reactions due partisanship or polarization (Molina et al. 2021). We thus devote a part of this study to political ads, investigating the ads dynamic around elections and other political events, such as Super Tuesday in May 2016, the Midterms in November 2018 and the government shutdown in December 2018. We also study potential distinctive impacts of political ads on readers' perception of news publishers.

Given the substantial role of these ad formats in the business model for journalism and evidence of declining trust in media and other institutions in the United States (Ladd 2012, Knight Foundation 2018) and elsewhere, there is a need for research quantifying their consequences.

\subsection{Related Literature}

The digital era dramatically impacted the business models of newspapers (Kirchhoff 2010, Cawley 2019). It particularly affected the advertising market for news media (Athey and Gans 2010, Athey et al. 2013), with consequences on the journalistic content and the readership (Angelucci and Cagé 2019). After Ellman and Germano (2009) hinted towards potential collusion between advertisement and media quality, empirical research found evidence that online publishing was, to some extent, shaped by advertisement programs (Sun and Zhu 2013, Seamans and Zhu 2014, Beattie et al. 2021).

Different ad formats emerged on websites, posing new questions about ads efficiency and interaction with organic content, which are typically ostensibly separated (cf. Beattie et al. 2021). While display ads have been extensively studied (Choi et al. 2020), the literature on native ads is limited. Additionally, native advertising formats - since they are designed to fit with the organic content are highly varied, including such varied formats as ads in general and place search results and the news-styled formats we consider (Wang et al. 2019). 
Researchers have seen great potential in native advertising (e.g., Becker-Olsen 2003, Ferrer Conill 2016, Abhishek et al. 2019) yet warned that clear sponsorship disclosure was key to avoid deceiving consumers (Bakshi 2014, Campbell and Marks 2015). Research on native ads includes studies on different types of native ads that all have in common that they blend in with organic content. We discuss hereafter investigations of various native ads type.

Studies based on large-scale randomized experiments found no evidence that labeled native ads on mobile search apps deceived the audience (Sahni and Nair 2020, Park et al. 2020). Yet, native ads' perception in other contexts has been subject to debate. For instance, research on search advertising - native ads that appear among "organic" search results - found no evidence of adverse reactions to targeted and labeled search ads in a field randomized experiment with 3.3 million U.S. users (Sahni et al. 2019). On the other hand, Cramer (2015) found that content-relevant search ads, even with high quality, decreased the perception of the overall listing quality. Furthermore, other scholars have raised concerns about how premium native ads - those that are deeply integrated into the editorial content of the news website and usually created with involvement from the publisher - deceive consumers, potentially impacting the publishers' credibility (Wu et al. 2016, Krouwer et al. 2017, Amazeen and Wojdynski 2019).

Besides empirical research, scholars have also theoretically motivated the need for disclosure in ads markets and its implementation through regulation (Milgrom 2008). Chatterjee and Zhou (2021) further detail equilibrium conditions for the use of native ads over traditional ads in a two-sided market.

While this past research mainly focuses on the effectiveness of native ad formats and consumers' perception of the advertisers, there is limited and mixed evidence on the effects of native advertising on trust toward the organic content it surrounds, and far less so on the impact of the ubiquitous "recommended content" format on news publishers. As pointed out by Sahni and Nair (2020), a practical limitation for such research is that ads "affect a small number of people" and large sample sizes are required to detect subtle signals (Lewis and Reiley 2014, Sahni 2015).

We are aware of only two studies that focus on native ads in online news websites. A survey experiment compared exposure to a single sports ad, which was either in a native or banner format; with 450 participants, no effects on publisher credibility was detected (Howe and Teufel 2014). Moreover, Aribarg and Schwartz (2020) conducted multiple studies to compare the effects of display versus native ads for mainstream brands on news publications, finding that native ads increase click-through rate while reducing brand recognition and perceived trustworthiness of the news website. More specifically, this study investigates both the conflict at the heart of advertisers' activity (between generating clicks and preserving brand recognition) and the trade-off publishers face (between attracting clicks that generate revenue and safeguarding credibility). In these studies, 
participants could navigate on a fictitious but functional website between articles and ads. The authors tested the effect of disclosing ads from six well-known advertisement brands promoted either as native or display ads. The ads' effects on publishers' credibility appeared only after multiple exposures to what was innocuous, non-political ads by major brands; with a sample size of 1,300 participants, and there were no significant effects on trustworthiness for a one-time exposure to native ads. ${ }^{2}$

Finally, Bashir et al. (2016) is, to the best of our knowledge, the only paper that investigates the same type of native ads as us (that is, in-feed promoted content that blend with the feed of recommendations for articles as "next read" at the bottom or on the side of an article from the host publisher). These ads are typically disclosed under a "recommended content" headline, in widgets where articles and ads are mixed. The widgets are handled by third-party companies such as Taboola and Outbrain, which hence control both the recommendation system from the host publishers and the ad placement on those same publishers. The authors collected an impressive amount of native ads headlines, studying both their origins and the extent to which the ads were disclosed (instead of blurred into the editorial content). They found that ad providers failed to prominently disclose the paid nature of their sponsored content and flagged the low quality of the ad domains. To do so, they looked at the Alexa rank of the domain to which the native ads were pointing, showing that the largest ad aggregator companies were displaying a "small number of reputable properties and a long tail of unknown properties." The authors further reveal that advertisers operate redirecting strategies, re-routing the readers from a disclosed domain to another one. Finally, this paper highlights that the ad content typically relates to "commercial offers related to financial services, penny auctions, and medical services," and points out that clickbait ads emerge from their data, leaving the analysis of the style and topics of these ads mostly qualitative.

We pick up the analysis of this native ad network where Bashir et al. (2016) left it, providing a systematic investigation of the ad themes and their editorial style. We do not focus on disclosing strategies or ads' landing domain but instead study the headlines' content and its heterogeneity across different publishers. While Bashir et al. (2016) studied the ads' content through Latent Dirichlet Allocation to infer different topics that the ads covered, we used supervised learning to first label ads' headlines iteratively. We use a simple Bayseian heuristic to label headlines based on a validated training set, unlike Rony et al. (2017) that relies on more sophisticated natural language processing techniques.

After this descriptive analysis of the ads' headlines, we turn to investigate their impact on readers' perception of publishers' credibility. While Aribarg and Schwartz (2020) used a fictional

${ }^{2}$ Note that comparing display ads and native ads relates to the Interactive Advertising Model (Rodgers and Thorson 2000) that predicted that great discrepancy in the ads' perception could arise from different ad formats. 
publisher and well-known ad brands, we used real publishers and real ads of various quality, and topics are drawn from the native ad network we study. The nature of our studies then greatly differ: we focus on the interaction between a pervasive low-quality ad network and the credibility of established publishers, while Aribarg and Schwartz (2020) study the conceptual impact of highquality native ads on a hypothetical publisher. We then characterize the heterogeneity of the ads' impact across multiple news websites. Further, because our survey experiment is not functional, we do not draw conclusions on the readers' activity once they click on the ads, but rather simply on their evaluation of publishers' ecosystem with different native ads conditions.

Finally, our paper relates to the literature on the measurement of news credibility (Strömbäck et al. 2020). Our approach differs from other research on media trust in that it does not assess the articles' professionalism after an in-depth reading. In our experiments, only a small part of the article is displayed to the participants, with the ads placed at the bottom of the article, where they typically are. We then assess the credibility of the article based on this treatment. By randomly assigning different articles and ad conditions to the publishers, we can indirectly measure the causal effect of ads on perceived publisher credibility. This design resembles the research on information credibility in short texts (such as posts on Facebook or Twitter) (Shariff et al. 2017) or on characteristics of the websites' layout that drive credibility (Castillo et al. 2011). We also have a broad measure of credibility. As Tseng and Fogg (1999) noted, "the literature varies on how many dimensions contribute to credibility evaluation." Past research used the words credibility (Shariff et al. 2017), trust and false (Castillo et al. 2011) or multi-factor scales (Meyer 1988, Flanagin and Metzger 2000, Yale et al. 2015, Wölker and Powell 2021) when evaluating credibility. Our scale includes five factors: credibility, trustworthiness, falseness, bias, and informativeness. We investigate these different dimensions that we ultimately compare and combine. Following past experiments (Castillo et al. 2011, Shariff et al. 2017), each factor is evaluated on a multi-point scale. Like previous work, our work relates to the relationship between prior familiarity with a publisher and trust towards it (Pennycook and Rand 2019, Epstein et al. 2020), for which we include the prominence of publications and individuals' familiarity with them in our statistical models.

\subsection{Contributions and Overview}

1.2.1. Prevalence Study First, we study the native ad ecosystem and provide insights about the ads' dominant topics and editorial style. We provide a detailed analysis of native ads on U.S. online news publications. We do this relying on a large data set of 1.4 million native ads. Over one million native ads were collected in 2016 by Bashir et al. (2016) from a collection of 500 publishers. The remaining 400,000 ads were collected between 2018-2019 from a collection of 39 publishers, that represented a combination of 23 mainstream publishers chosen from Amazon's Alexa 2018 
ranking for top news websites and 16 publishers randomly chosen from the set of 500 publishers collected by Bashir et al. (2016). In both scraping schemes, an automated script was constantly running, cycling through the different websites and scraping multiple times the content of a random set of articles found on that website.

We chose to focus on the concept of clickbait ads in order to test the common concerns over the prevalence of such announcements on publishers' websites (Bashir et al. 2016). A total of 10,000 random ad headlines were classified through Mechanical Turk tasks $(n=1,073)$, where participants assessed whether the ad headlines were clickbait, according to the following definition given to them: "clickbait is something (such as a headline) designed to make readers want to click on a hyperlink especially when the link leads to content of dubious value or interest." We further discuss the clickbait construct in section 2.2. Given that both data gatherings occurred during elections, we chose to study the prevalence ads related to political events in the ad network. MTurkers were asked in the previously-described tasks to also choose up to two topics describing the ad. The prevalence of topics was then estimated for the entire set of ads using supervised machine learning methods.

The descriptive analysis reveals that clickbait ads constitute the large majority of the native ads and that politics is one of the most common topics in such ads, despite the fact that these ads are meant to promote products rather than a political agenda. We advance the literature by providing, to the best of our knowledge, the largest-scale quantitative study about the different ad types present in the dominant native ad network on news publishers.

1.2.2. Randomized Experiment Due to the prevalence of clickbait and political ads, we evaluate the causal effects of these kinds of native ads on publishers' credibility, creating hundreds of stimuli that replicate real articles surrounded by sponsored ads. ${ }^{3}$ Our experiment with large sample size $(n=5,040)$ and partially within-subjects (i.e; mixed) experimental designs allow us to average over many randomized stimuli (publishers, articles, ads). Our stimuli consisted of a publisher associated with randomized article and ad conditions. The ad conditions were: clickbait and non-political, clickbait and political, non-clickbait and non-political, non-clickbait and political, and no ad (the control condition). See Figure 3 for the details of the creation of the stimuli. Importantly, each publisher appeared paired with every article to fully control for article-specific effects. We tested eight publishers associated with one of eight articles, and each participant rated six stimuli featuring each distinct publishers and articles, and all five ad conditions (the control condition always appeared twice). Participants were asked to rate the credibility of the articles they

${ }^{3}$ Note that the articles were chosen to be timely but non-controversial to reduce article-specific effects and enhance a balanced selection of articles across sources. 
read, and not the publisher. However, the same article would appear under all possible publishers since we fully randomized the article with which a publisher was paired. Our experimental design allows to indirectly measure the effects of the ad conditions on the perceived credibility of different publishers.

The experiments provide evidence that even a one-time exposure to native ads can negatively impact readers' perception of publishers' credibility. Importantly, we do not find that the native ad format is problematic per se. Instead, clickbait and political ads, which are prevalent in the network, are responsible for the negative effects we observe. Articles hosting non-clickbait ads are statistically indistinguishable from articles without ads. Lower familiarity publishers - those publishers for whom less than $50 \%$ of the audience is familiar - drive the overall results.

We advance the literature by by quantifying the impact of native ads on the credibility of news publishers they border. Unlike Aribarg and Schwartz (2020), we do not compare display ads versus native ads, but we uncover different kinds of native ads that are perceived differently under the same disclosure style. Our work informs the need for detailed regulation of native ads, and suggests that native ads platforms might need to invest more in self-regulation of the content they gather and display. It further supports publishers in making informed decisions about their advertising choices.

The rest of the paper is organized as follows. Section 2 presents a descriptive study of the content of native ads displayed by leading publishers. Section 3 presents the randomized experiment in which we varied ads, articles, and publishers. Section 4 concludes, discusses further research and the policy implications of our work.

\section{Prevalence Study}

We begin by examining the prevalence of native advertising - and describing its content - alongside editorial content from leading news publishers in the United States. We document the widespread prevalence of clickbait and political ads.

\subsection{Data}

We used two data sets: (i) a 2016 data set collected by Bashir et al. (2016), that contains information on one million ad headlines published across 500 publishers between February 26 and March 4, 2016, and (ii) 2018-19 data set that we collected using the techniques of (Bashir et al. 2016) which contains 400,000 ad headlines published on 39 publishers. We established the list of publishers through a combination of 23 mainstream publishers listed on Amazon Alexa's news websites rankings ${ }^{4}$ and

\footnotetext{
${ }^{4}$ The ranking used to be available at ttps://www.alexa.com/topsites/category/News, but was retired on September 2020. We selected all the websites that hosted at least one native ad widget, and that was not a weather or news aggregator website as of August 2018.
} 
16 publishers randomly sampled from the set of 500 publishers in the previous work (Bashir et al. 2016)) between November 1, 2018 and February 2, 2019. We varied the IP address of the machine scraping the data in order to account for potential targeting algorithms serving different ad types in different locations. We randomly changed the IP address of the scraper to have a representative sample of displayed ads based on the following locations: Boston, Seattle, Houston, San Jose, New York, Miami, Atlanta, and Cambridge (Appendix 1.2.2). For each of the 39 chosen publishers, we scraped the ad widgets (where native ad companies display the ads thumbnail) contained in the publishers homepage in addition to 20 randomly selected articles. Moreover, we set the scraper code to scroll each page five times each day to cover multiple appearances of ads.

Combined, this constitutes 1.4 million native ad headlines. Note that by nature of these ad widgets, they host a mix of third-party ads and recommended articles from the host publisher. We differentiates these two types comparing the destination url with the website's (Appendix 1.1.1).

Finally, the first dataset was collected in 2016 during the U.S. primary elections and the latter during four months, starting a couple of days before the 2018 U.S. midterm elections. The timing of our data collection allowed us to further study the display of topical political ads in the ad network. We labeled the ads based on their topic and their editorial style, and provide a descriptive analysis of the various topics present in the ads, with an emphasis on political ads. Indeed, political ads signal an interesting marketing dynamic we believe requires further attention. Additionally, we provide a quantitative measure of the ad style, which we capture under the concept of clickbait described hereafter.

\subsection{Clickbait construct}

We use the concept of clickbait to describe the editorial style of some of native ad headlines. Content is qualified as clickbait if "it is designed to induce readers to click on it, especially when it leads to content of dubious value." ${ }^{5}$. For instance, "Mycha started drinking two glasses of bittergourd juice every day for seven days and the results are amazing" or "So you bought someone a gadget. Here's how not to become their tech support" are clickbait headlines. Clickbait headlines "are frequently used by both legitimate and less-than-legitimate news sources" (Munger et al. 2020). Hence, without additional context, the veracity and the real intent of an ad's headline are uncertain. Advertisers use the clickbait style and "appear to be generally deceptive or misleading" (Rey 2013). Yet, clickbait needs not to be a proxy for the quality or professionalism of the content. In fact, "So you bought someone a gadget. Here's how not to become their tech support" is a headline from the New York Times that accurately describes the article's content. Hence to assess

${ }^{5}$ As per Merriam-Webster's definition, see https://www.merriam-webster.com/dictionary/clickbait. This definition has been now used in multiple academic works including Zhou (2017), Shinkhede (2019), Sisodia (2019), Banerjee and Urminsky (2022), and Munger (2020). 
whether a headline is inaccurate or misleading, the source and the content are probably better indicators than the style.

However, a growing body of research (Kalveks 2007, Bazaco 2019, Munger et al. 2020, Munger 2020) focuses on the use of clickbait style in online content and signals that there is a need to "[monitor] its consequences on the quality of the news circulating on social networks" (Bazaco 2019). Following this line of thought, we evaluate the widespread assumption under which which native ads contain many clickbait headlines - and we further use this classification in subsequent experiments.

\subsection{Labeling schemes}

In this section, we present the methodology used to label ad headlines.

2.3.1. Unsupervised and supervised labeling methods Herein, we specify how we constructed the labels, that is the topic categories and the binary clickbait classification. We inferred the main ad topics using latent Dirichlet allocation topic modeling (Blei et al. 2003), and refined the topic selection with a Mechanical Turk task $(\mathrm{n}=139)$, resulting in the following topic dictionary: politics, education, personal finance, healthcare, technology, sports, entertainment, culture/religion, romance/dating, retail, local news, and other. We then used Mechanical Turk tasks (n $=1,073)$ to label 10,000 random headlines (5,000 from the 2016 data and 5,000 from the 2018-2019 data). Labellers first chose up to two topics describing the ad headlines and then assessed whether the ad headlines were clickbait. We provided the respondents with our definition of clickbait (see 2.2 ) at the beginning of the survey, and subsequently asked about each headline: "According to what you read, do you believe the headline is clickbait?" 6 Each headline was labeled on average 10 times. We expanded the labeling to the rest of the headlines using a binary Bayes classification, running separate analyses on the 2016 and on the 2018-2019 data (see Appendix 1.2.1 for distribution of topics). Namely, using the labeled data, we estimated the conditional probability of appearance of a word given the headline's label (political or clickbait). Then, we computed the posterior probability of a headline being political (or clickbait) conditioned on the set of words it contained (see Appendix 1.1.2 for more details).

2.3.2. Robustness checks on labeling Given that each headline was labeled multiple times, we compute the inter-reliability scores (metric to describe the coherence of the different rating collected) to quantify the level of agreement among different workers. As each worker labelled a different subset of the entire headlines in the training test, we use the generalized Fleiss's Kappa

${ }^{6}$ Note that the respondents performed the classification task without the source of the headlines. This procedure, therefore, classifies clickbait headlines from legitimate and less-than-legitimate sources under the same category. Thus, we cannot assume that our clickbait measure is a measure of quality. Instead, it is an editorial style designed to entice clicks, which may nonetheless be correlated with quality. 
statisitc (Gwet 2014). We find that the inter-reliability scores are: 0.646 for the clickbait category for the 2016 data, and 0.619 for the 2018 data, 0.897 for the political category in 2016, and 0.899 in 2018. It is unsurprising that workers agree more on the well-established political category than on the more subjective clickbait category. These agreement rates are considered as substantial agreement for the clickbait category, and as almost perfect agreement for the political category (Landis and Koch 1977).

Further, we test for the external validity of the classification of all the headlines in the test set: over $80 \%$ of the headlines were successfully classified within at least one topic, and the crossvalidation scores per classifier were between $92 \%$ and 99\%. See Appendix 1.1.2 for more details about the confusion matrices and the words associated with each topic.

\subsection{Results}

Clickbait headlines were widespread, constituting $82 \%$ of the headlines set. Interestingly, a comparable percentage of headlines across topics were found to be clickbait. Hence, themes that are intrinsically clickbait, such as entertainment, were not responsible for the overall proportion of clickbait ads. As we analyzed prevalent features that described clickbait headlines, we found that the features that differentiate clickbait ads from ads that are not clickbait are the use of particular words and the use of certain forms of punctuation. For instance, clickbait ads call out the reader. In fact, the word "you" is used 7.5 times more often in clickbait ads than in non-clickbait ads, such as in "[Gallery] Stars You May Not Know Passed Away." Furthermore, clickbait headlines use nearly twice as much active verbs such as see, can, look or do than non-clickbait headlines, such as in "Trump successes are boosting GOP candidates in midterms - Don't expect a blue wave." Finally, clickbait-style contains nearly three times more punctuation $(?, !$, and ...) than non-clickbait style.

The prevalence analysis found that $11 \%$ of the headlines were political. The politics topic ranked third out of twelve, behind entertainment (34\%) and personal finance (13\%). Further, consistent with the reputation of native ads to make use of trending topics, we found that headlines featuring Donald Trump in 2016 were common. ${ }^{7}$ Further, we observed that native ads were more politicized during political events. Indeed, reporting the temporal evolution of content in native ads between November 2018 and February 2019, we found that the amount of political ads was higher during the midterms and the government shutdown than around Christmas (see Appendix 1.2.3). ${ }^{8}$

In addition, since the ad brokers are in charge of many different publishers, we were interested in quantifying the extent to which the ads served per publisher are tailored to that specific publisher.

${ }^{7}$ During the 2016 campaign, headlines containing the words "Trump" or "Donald" in the ad headlines were three times more frequent than headlines containing the words "Hillary" or "Clinton."

${ }^{8}$ Note that the political ads we encountered manually were rarely associated with well-known, reliable or high-quality sources. 
Figure 2 Prevalence of clickbait and politcal native ads among publishers.
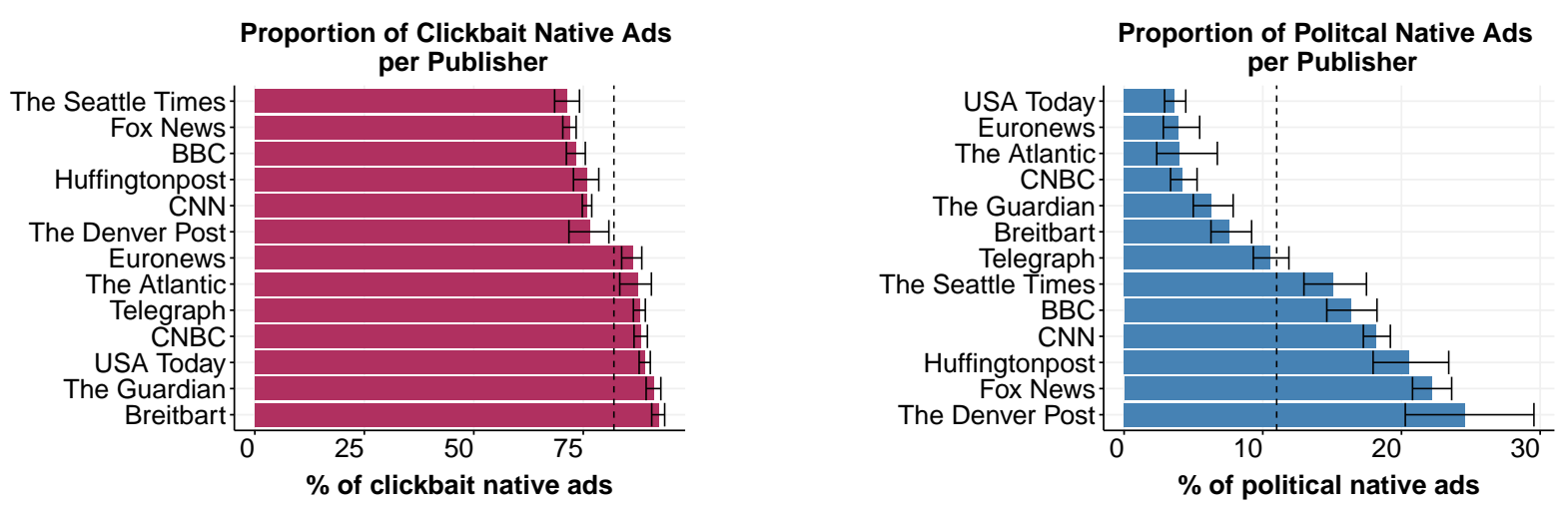

Note. Clickbait (left) and political (right) proportions with 95\% confidence intervals in native ads per publisher. dashed line represents average over 500 publishers under study. These ad widgets incorporate a mix of third-party ads and recommended articles from the host publisher. All of the scraped headlines were labeled, regardless of whether they were ads or articles. However, this plot only shows the clickbait and political rates in third party native ad headlines.

We found that different publishers host significantly different proportions of political $\left(\chi_{12}^{2}=926\right.$, $\left.p<10^{-15}\right)$ and clickbait $\left(\chi_{12}^{2}=873, p<10^{-15}\right)$ ads, consistent with the idea that different publishers host different type of ads. Figure 2 displays this heterogeneity among 13 publishers. Further descriptive analyses can be found in Appendix 1.2.

\section{Randomized experiment}

Given the widespread use of these native ad formats by news publishers - and the prevalence of clickbait and political headlines in the ad content, we hypothesize that there could be substantial negative effects of the presence of these ads on assessments of the credibility of news publishers. We sought to test for such effects through an experiment where we systematically vary ad content, articles, and publisher brands. Our experiment focuses on a widespread everyday experience: skimming through the beginning of a news article that is accompanied by ads styled as recommended related articles under discrete real-world disclosure. Scanning headlines and skimming through articles have become a common way to consume news (Liu 2005). Thus, the "first-sight assessment" of news publishers has become increasingly important, and online publications need to showcase an attractive yet reliable environment to retain readers. We, therefore, hypothesize that the presence of dubious inappropriately-labeled native ads alongside articles readers skim through can affect online publications' appearance, subsequently hurting their credibility. To simulate such context, our survey was designed such that respondents could only read first few paragraphs of the news followed by a "Read more" button. However, the experiment does not fully emulate a real experience, since respondents could not freely explore the publishers' page to choose articles, or 
click on the following ads. This experiment was informed by the result from our pre-experiment which is fully outlined in Appendix 2. Furthermore, the design and analysis methods used were pre-registered prior to running the experiment. The pre-registration plan can be found in Appendix 3.5 .

\subsection{Credibility scale}

3.1.1. Definition and function of the credibility scale Our key dependent variable in the specification used to highlight causal effects of native ads is an overall credibility score. We asked participants to assess each article on five factors related to the credibility of the source material trustworthiness, credibility, newsworthiness, falseness, and bias - on a five-point agree-to-disagree scale (i.e. a Likert-type scale), consistently with previous measures (Castillo et al. 2011, Shariff et al. 2017).

Our focus is on the reader's high-level assessment of an article when skimming through the news since this activity arguably represents an important portion of readers' interaction with news websites (Wolf 2018). Our approach differs from the literature on media trust in that it does not relate to assessing articles' credibility, authenticity or balance, nor does it ask participants to rate the journalistic intent when selecting news or differentiating factual from opinionated articles. (For an overview of measuring media trust, see Strömbäck et al. (2020)).

We adapt the literature's methodology (Meyer 1988, Flanagin and Metzger 2000, Castillo et al. 2011, Shariff et al. 2017) to fit our research purposes, formulating our assessment task as follows (note that the order of the questions was randomized):

Please rate the extent to which you agree or disagree with the following descriptions of the article.

- The article was false.

- The article was biased.

- The article was trustworthy.

- The article was credible.

- The article provides news information.

3.1.2. Robustness checks on the credibility scale We evaluated the psychometric properties of this credibility scale. As expected, the five items (trustworthiness, credibility, newsworthiness, falseness, and bias) were highly correlated (Appendix 3.4.1). Principal component analysis (PCA) and exploratory factor analysis indicated that the five items tapped a single dimension (Appendix 3.4.2). The Cronbach's $\alpha$ of these five items was 0.793 (with a 95\% CI of [0.788 0.798] computed by 1,000 bootstrap replicates) which indicates the internal consistency of the items. Throughout we use the average score across the items as our dependent variable (negative measures 
Table 1 Publishers' Familiarity Classification.

\begin{tabular}{c|c|c} 
Familiarity Score & Classification & Publishers \\
\hline$>75 \%$ & High & CNN, Fox News, USA Today \\
\hline$[25 \%, 50 \%]$ & Medium & The Atlantic, The Denver Post \\
\hline$<25 \%$ & Low & Sacramento Bee, Daytona Beach, Vox \\
\hline
\end{tabular}

The eight publishers in the experiment were chosen based on an independent survey $(\mathrm{n}=1,514)$ assessing familiarity with 20 news publishers. Based on the results of our pre-experiment, we expected the treatment effect to vary based on publishers overall familiarity level. As a result, we selected eight publishers that represented different segments of the familiarity distribution as shown in the table. Note that during the experiment, we also measured familiarity for a set of publishers including the eight publishers that were used to create the stimuli. In this sample, $30 \%$ of the participants surveyed in Experiment 2 were familiar with Vox, hence we analyzed both classifications - Vox as low familiarity as pre-registered, and Vox as medium familiarity as a post-hoc analysis (see Appendix 2.2.3).

- falseness and bias - were reversed). To put this on a more interpretable scale, the credibility score is defined as the averaged score normalized by the standard deviation of the average ratings in the experimental condition without native ads.

\subsection{Experiment}

We use a sophisticated design that samples stimuli randomly from all publisher-article pairs to allow controlling for article-specific effects and estimation of the causal effect on publishers' credibility. Furthermore, we systematically varied different ad conditions using the labeled data from the prevalence study. The experiment tests a wide range of treatments (clickbait, non-clickbait, political clickbait, political non-clickbait, and no ad).

3.2.1. Participants We registered a target sample size of 5,000 US residents recruited via the survey platform Dynata. In total, 7,669 participants began the survey; and 5,040 completed the survey. We retained all individuals who finished the study $(\mathrm{n}=5,040 ;$ mean age $=47 ; 55.3 \%$ women). 14 respondents who did not rate any article were removed from the sample. Note that participants provided informed consent and the study was approved by the university Committee on the Use of Humans as Experimental Subjects.

3.2.2. Materials As shown in Figure 3, 320 publisher-article-ad pairs $(8$ publishers $\times$ eight articles $\times 5$ treatment conditions) were created semi-automatically. First, the HTML code for the ad sets was generated. Then, templates were created for each eight publishers format, allowing to fix text length and to standardize the environments, and HTML code for the eight articles, and the five ad conditions were inserted in the publishers' templates. Samples of the stimuli can be found in Appendix 3.1.1)

Informed by the results from the pre-experiment, which suggested that the significant effects were heterogeneous across publishers, in the main experiment we tested a wider range of ads and publishers. We hypothesized that the overall readers' familiarity with publishers could be a proxy 
for various characteristics causing the heterogeneity of effects. Hence, before conducting Experiment 2 , we did an independent survey $(\mathrm{n}=1,514)$ assessing familiarity with 20 news publishers and selected eight of them that represented different segments of the familiarity distribution (Appendix 3.1.2). Table 1 shows the lists of publishers and their classification criteria. More precisely, we chose three publishers scoring above $75 \%$ in familiarity rate (CNN, Fox News, and USA Today), two publishers scoring between 25 and 50\% (The Atlantic and The Denver Post) and three publishers scoring below $25 \%$ (Vox, Sacramento Bee, and Daytona Beach). ${ }^{9}$

Two distinct batches of ad sets were created and randomly matched with article-publisher pairs, to partially account for ad-specific effects. Each batch is constituted of four sets of four ads, each set representing one of the four treatment conditions. Clickbait and political ads were chosen based on the clickbait and political scores assessed by the classifier trained on the prevalence data. ${ }^{10}$

3.2.3. Procedure Participants were asked a series of questions measuring demographics, need for cognition, and media habits. Attention check questions were distributed across the survey. ${ }^{11}$ Each participant read a random and balanced selection of six articles paired with different publishers and ad sets. Namely, the six stimuli each consisted of a distinct article and publisher; two stimuli appeared under the no-ad condition and the four other stimuli each appeared under one of the remaining four treatment conditions. Before reading the articles, respondents were faced with the following prompt:

You will find the first article on the next page. Please read it carefully before answering the following questions. There will be a brief pause on the each screen with an article so you can read the entire story. At the end of the pause, an arrow will appear at the bottom of the screen. Once the arrow appears, you may move to the next screen of the survey by clicking on the arrow. We are interested in knowing what you were thinking about while you read the article. You might have had ideas all favorable to the article or author of the article, all opposed, or a mixture of the two. Any case is fine; simply answer those questions that appear after the articles according to your thoughts.

After exposure to the stimulus, participants rated the article they read on the five-item credibility scale 4. In order to avoid demand effects, we also asked respondents to answer an article-specific

${ }^{9}$ In the experiment, we also measured familiarity for a set of publishers including the eight publishers that were used to create the stimuli. In this sample, $30 \%$ of the participants surveyed in Experiment 2 were familiar with Vox, hence we analyzed both classifications - Vox as low familiarity as pre-registered, and Vox as medium familiarity as post-hoc analysis (Appendix 3.4.6).

${ }^{10}$ Because the labeling was done on the headlines alone - without images -, we ran an internal test to validate the choice of the ads in each ad set. To do so, we selected ads that had the highest political, non-political, clickbait and non-clickbait scores and set up an internal survey where each of us five classified the ads' thumbnail (with headline and associated image). We finally constituted the ad sets based on this validated ad classification.

${ }^{11}$ Three attention checks measured whether participants were reading the instructions and not clicking randomly. In the beginning and the middle of the survey, we asked participants to choose two given options among a set of six and 18 options respectively. The last attention questions were grids containing six general knowledge or opinion questions. See Appendix 4 for the full list of survey questions. 
Figure 3 Creation of stimuli pairing each publishers with all articles and ad conditions.

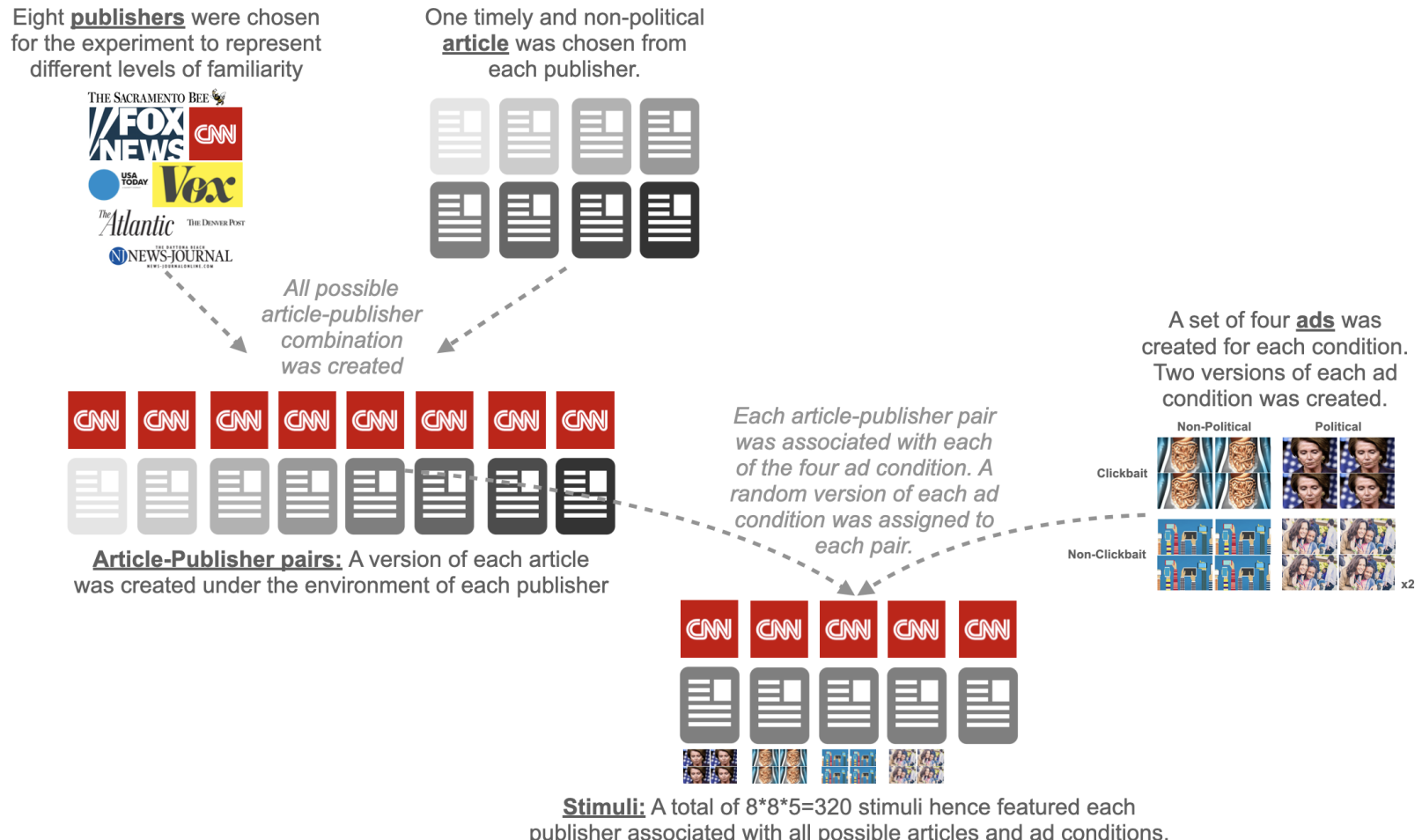

Note. There are eight publishers under study: CNN, Fox News, USA Today, The Atlantic, The Denver Post, Vox, Sacramento Bee, and Daytona Beach (top left). A timely and non-political article was chosen from each publisher (top middle). There are five ad conditions under study (top right), one control (no ad) and four treatments (clickbait non-political ads, clickbait \& political ads, non-clickbait ads and non-clickbait \& non-political ads). Each treatment condition is a set of four ads chosen systematically. We created two versions of each treatment condition to account for ad-specific effects. A version of each article was embedded in each of the eight publishers' ecosystem (bottom left). These choices allow to control for article-specific effects. Finally, each publisher-article pair was combined with all five ad conditions (bottom right); one out of the two versions of the ad set was chosen at random to be paired with each publisher.

distractor question related to each article's content before moving to the credibility questions. We decided to expose each participant to six stimuli following the results of a power analysis on the preexperiment we conducted. We concluded that six stimuli with a sample size of 5,000 respondents provide us with more than $75 \%$ power to observe half the effect size observed in the pre-experiment. Details on power analysis procedure can be found in Appendix 2.7.

Note that, by design, the stimuli contain only the first paragraphs of the articles, and respondents could not read the full piece. The reading is purposefully limited in order to avoid the participants focusing on details in the actual content. Further, we deliberately chose benign news articles so that the article content does not drive the perception of the article.

3.2.4. Analysis Strategy The credibility scale was measured by averaging the five items per article, after reversing bias and falseness ratings. Mean imputation was performed on 506 out of 
Figure 4 Randomization procedure of the stimuli

Each participant was exposed to a random selection of six unique publishers out of the eight publishers under study. Each publisher was paired with a distinct random article. Further, each treatment condition appeared once, and the control appeared twice.
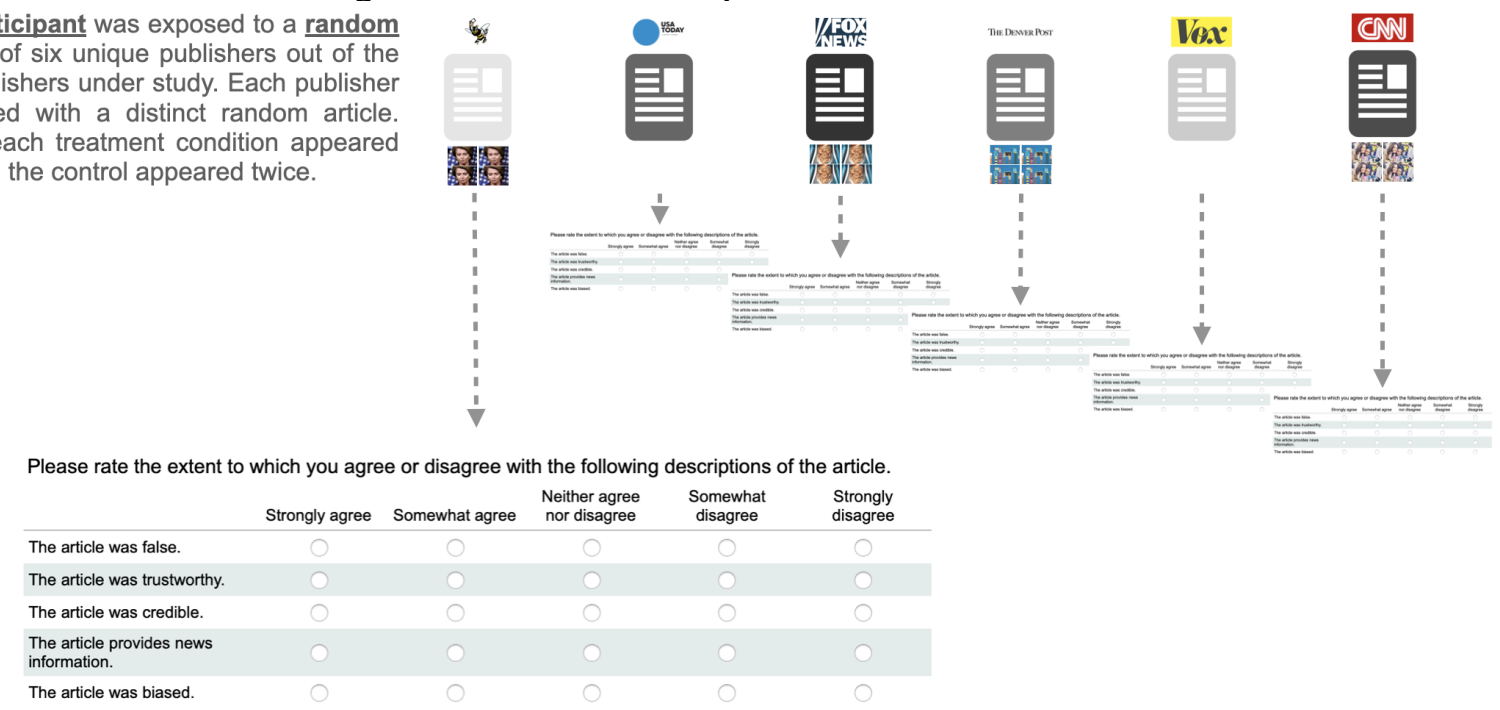

After a distraction question about the content of each piece they had read, the participants were asked to rate the article based on the five following factors.

Note. Each participant was exposed to six distinct publishers paired with six distinct articles, and to all five ad conditions (once for each treatment condition, and twice for the control condition). To prevent priming the participants about the goal of our study, we asked an article-specific question before requiring them to rate the articles based on five credibility factors (bottom).

30,156 ratings. The score was normalized by the standard deviation of the mean response to stimuli with no ads (0.806). Estimates of average treatment effects $(\widehat{A T E})$ for different ad contrasts were computed using a mixed effects model (R, lmer). Random effects were added at the participant, publisher, and article levels. The following covariates were added to the model to improve the inference precision: age, gender, education, cognition scores, individual familiarity with publishers, political leaning, political interest, ad noticed, attention score, and aggregated familiarity with publishers. Mean imputation was also performed on covariates when needed, except for political interest which was based on a unique question, hence the missing values were treated as a "did not express an opinion" category.

The mixed effects model used for esimation of $\widehat{A T E}$ is formulated as:

$$
y_{i, p, a}=\alpha_{i}^{i n d}+\alpha_{p}^{p u b}+\alpha_{a}^{a r t}+X_{i, p, a}^{T} \gamma+\sum_{t \in \mathcal{T}} \mathbb{1}_{\left\{T_{i, p, a}=t\right\}} \beta_{t}+\epsilon_{i} .
$$

And the following model estimates the heterogeneous treatment effects across publishers' aggregated familiarity levels using interaction with the treatment:

$$
y_{i, p, a}=\alpha_{i}^{i n d}+\alpha_{p}^{p u b}+\alpha_{a}^{a r t}+X_{i, p, a}^{T} \gamma+\sum_{t \in \mathcal{T}} \sum_{k=1}^{3} \mathbb{1}_{\left\{T_{i, p, a}=t\right\}} \mathbb{1}_{\left\{L_{p}=k\right\}} \beta_{t, k}+\epsilon_{i} .
$$


where $y_{i, p, a}$ is the credibility score given by individual $i$ to the pair (publisher $p$, article $a$ ), $\alpha_{i}^{i n d}, \alpha_{p}^{p u b}$ and $\alpha_{a}^{a r t}$ are random-effects for the participants, publishers and articles respectively. Note that since we randomized pairing of publishers and articles in the experiment, we could add separate random-effects for each. $X_{i, p}$ is a vector of covariates including age, gender, education, cognition scores, individual familiarity with publisher $p$, political leaning, political interest, a dummy variable for noticing the ad, attention score (drived from attention check questions), and aggregated familiarity level of publisher $p$ (based on Table 1). Even though the treatment assignment is randomized, including covariates can theoretically improve the inference precision. However, in practice, adding them did not make much difference in estimated standard errors since they were balanced across treatment conditions (see SI section 2.1.8 for balance tables). $T_{i, p, a}$ is the treatment variable which, depending on the contrast of interest, takes values in the set $\mathcal{T}=\{$ political,non-political $\}, \mathcal{T}=\{$ political,no ads $\}, \mathcal{T}=\{$ clickbait,non-clickbait $\}$, or $\mathcal{T}=$ $\{$ clickbait, no ads $\} . L_{p} \in\{$ Low, Medium, High $\}$ is a categorical variable identifying the aggregated familiarity level of publisher $p$ (based on Table 1). Finally, $\beta_{t}$ is the average treatment effects and $\beta_{t, k}$ the heterogeneous treatment effect for publisher familiarity level $\mathrm{k}$ and treatment condition t. Summary of the estimated treatment effects can be found in Table 2. The full regression table containing all covariates' coefficients is reported in Appendix 3.4.4. Also, Appendix 3.2.2 provides balance tables for the mentioned covariates.

We used random effects at the individual and publisher levels since it is more efficient than fixed effects. The random effects model does not discard the between-subjects variation and hence results in more precise estimates (Firebaugh et al. 2013, Greene 2003). Fixed effects are usually preferred in observational studies because the inclusion of random effects can cause omitted variable bias. However, in our setting the treatment assignment is completely random with respect to individuals, publishers, and articles, so fixed effects are not required for identification. Although inclusion of random effects causes some bias in estimating other covariates' coefficients $(\gamma)$, we are not making causal claims about those parameters. This model also more efficiently exploits our experimental design which has both within- and between- subjects variation. The within-subjects variation is due to the fact that each person observes publisher-article pairs with and without native ads. But we also have between-subjects variation since publisher-article pairs containing ads randomly change across individuals.

In our pre-registrated analysis plan, we submitted a three-way interaction model which included the interaction with individual familiarity indicator, as well as as the aggregate publisher familiarity. This pre-registered model, with properly defined test-statistic, can also be used to recover results very similar to those in Table 2. To calculate significance for any given comparison, a two-sided Wald test or a t-test were performed on the estimated treatment effects. (See Appendix 3.3 for details on alternative analysis strategy and mixed effect models in use.) 
Table 2 Impacts of native ads on publishers' credibility.

\begin{tabular}{|c|c|c|c|c|}
\hline & \multirow[t]{3}{*}{ Overall Model } & \multirow{2}{*}{\multicolumn{3}{|c|}{$\frac{\text { Aggregated Familiarity Model }}{\text { (Publisher Familiarity })}$}} \\
\hline & & & & \\
\hline & & (Low) & (Med) & (High) \\
\hline CB vs. No-ads & $\begin{array}{c}-0.024^{* *} \\
(0.011)\end{array}$ & $\begin{array}{l}-0.020 \\
(0.019)\end{array}$ & $\begin{array}{c}-0.050^{* *} \\
(0.024)\end{array}$ & $\begin{array}{l}-0.010 \\
(0.019)\end{array}$ \\
\hline $\mathrm{CB}$ vs. Non-CB & $\begin{array}{c}-0.023^{*} \\
(0.011)\end{array}$ & $\begin{array}{l}-0.020 \\
(0.019)\end{array}$ & $\begin{array}{c}-0.061^{* *} \\
(0.024)\end{array}$ & $\begin{array}{l}-0.003 \\
(0.019)\end{array}$ \\
\hline Pol vs. No-ads & $\begin{array}{c}-0.024^{* *} \\
(0.011)\end{array}$ & $\begin{array}{l}-0.026 \\
(0.019)\end{array}$ & $\begin{array}{c}-0.040^{*} \\
(0.024)\end{array}$ & $\begin{array}{l}-0.010 \\
(0.019)\end{array}$ \\
\hline Pol vs. Non-Pol & $\begin{array}{c}-0.022^{* *} \\
(0.011)\end{array}$ & $\begin{array}{c}-0.032^{*} \\
(0.019)\end{array}$ & $\begin{array}{c}-0.041^{*} \\
(0.024)\end{array}$ & $\begin{array}{c}0.001 \\
(0.019)\end{array}$ \\
\hline $\begin{array}{l}\text { Publisher Familiarity } \\
\text { interaction included }\end{array}$ & No & Yes & Yes & Yes \\
\hline Note: & & ${ }^{*} \mathrm{p}<0.1$ & ${ }^{* *} \mathrm{p}<0.05$ & ${ }^{*} \mathrm{p}<0.01$ \\
\hline $\begin{array}{l}\text { The summary of the ar } \\
\text { the models in equations } 1 \\
\text { No-ads, Clickbait vs. Non } \\
\text { Non-Political ads. The re } \\
\text { from non-clickbait or non } \\
\text { exposure to Clickbait/Pol } \\
\text { credibility of the publisher } \\
\text { lishers. }\end{array}$ & $\begin{array}{l}\text { rage and heteroge } \\
2 \text {. Rows correspo } \\
\text { Clickbait, Political } \\
\text { ults show that no- } \\
\text { olitical ads. Also, } \\
\text { tical ads has a neg } \\
\text { where the effect is }\end{array}$ & $\begin{array}{l}\text { eous treatr } \\
\text { ad to differe } \\
\text { ads vs. No- } \\
\text { ads are stat } \\
\text { regardless o } \\
\text { ative effect } \\
\text { strongest fo }\end{array}$ & $\begin{array}{l}\text { ent effects, e } \\
\text { t contrasts: } \\
\text { ds, and Poli } \\
\text { stically indis } \\
\text { the contrast } \\
\text { n readers' p } \\
\text { medium fam }\end{array}$ & $\begin{array}{l}\text { timated by } \\
\text { lickbait vs. } \\
\text { cal ads vs. } \\
\text { inguishable } \\
\text { a one-time } \\
\text { rception of } \\
\text { liarity pub- }\end{array}$ \\
\hline
\end{tabular}

3.2.5. Average treatment effects As summarized in the first column of Table 2, experiment results show that a one-time exposure to clickbait and political ads on average has a significant negative impact on credibility of publishers. At the aggregated level, clickbait ads decreased credibility, independently of whether one compares the effect of clickbait ads versus no ads or the effects of clickbait ads versus non-clickbait ads. In fact, non-clickbait ads were statistically indistinguishable from no ads. Further, political ads also reduced publishers' credibility, independently of whether one compares the effect of political ads with no ads, or the effects of political ads vs. non-political ads.

We made additional comparisons to put these effects into perspective, and interpret the extent of negative impact of clickbait or political ads. For example, the main effects for the aggregated publishers are almost two-fifths of the difference between $C N N$ and Fox News average ratings, a third of the maximum difference between average ratings among all publishers, and a ninth of the difference between Republicans and Democrats average ratings. These results indicate that native ads pose a threat to publishers' credibility when these ads are clickbait or political, while publishers with non-clickbait or non-political ads are indistinguishable from publishers without ads.

3.2.6. Heterogeneous treatment effects These overall negative effects appear to be driven by medium and low familiarity publishers. As shown in the second to fourth columns of Table 
Figure 5 Summary of the result for the experiment (left) and prevalence study (right)
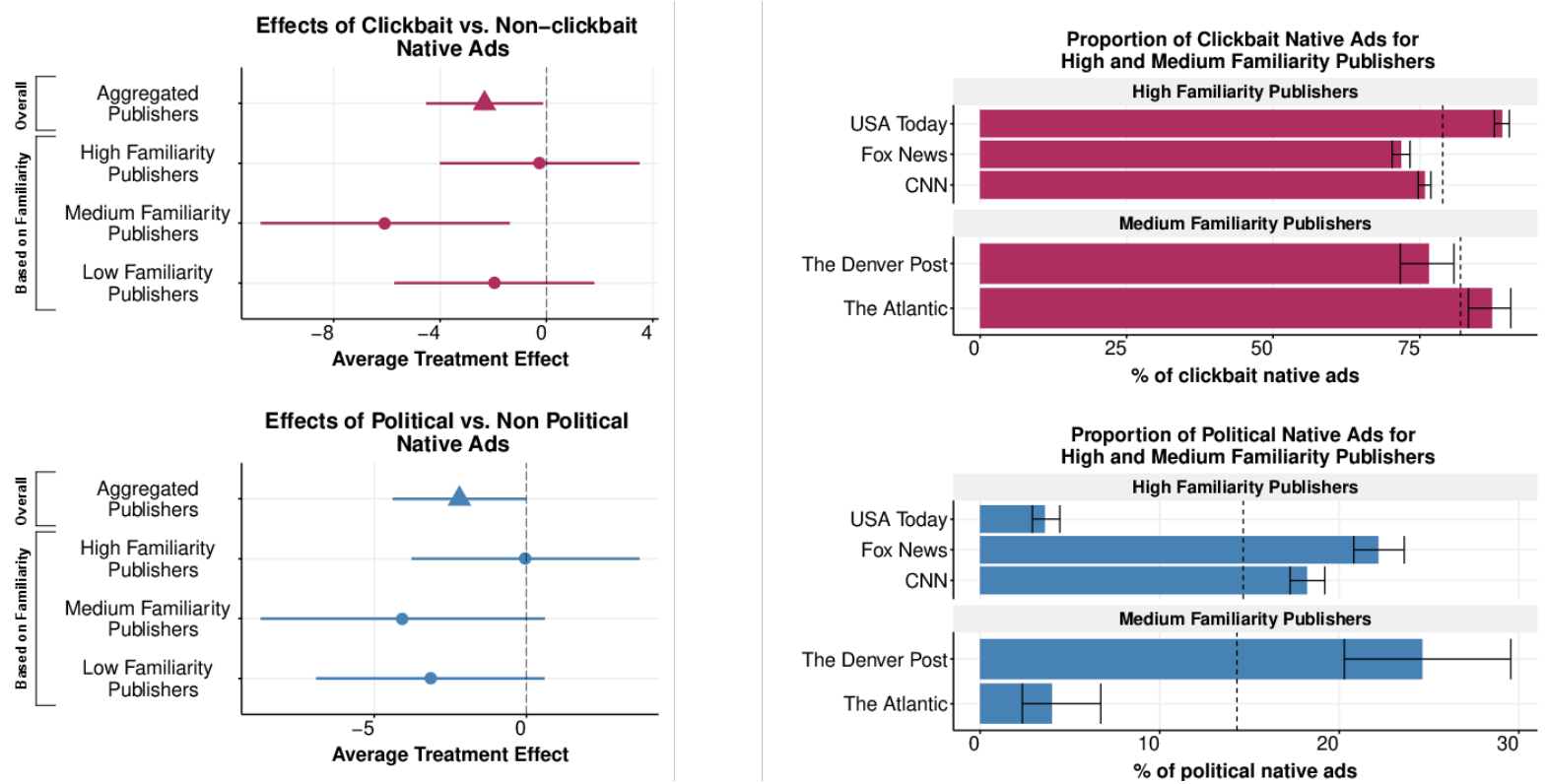

Note. Average treatment effect from Experiment 2 (left) and proportion (right) of clickbait (top) and political (bottom) ads per publishers grouped by familiarity level. Low familiarity publishers are not included in the prevalence analysis, so lack clickbait and political scores. The left plots show the average treatment effects multiplied by 100 of clickbait (top) and political (bottom) ads and 95\% confidence intervals. The right plots show the clickbait (top) and political (bottom) percentage in each publisher with 95\% confidence intervals. Dashed lines represent the average score for publishers displayed on the plot.

2, clickbait and political ads significantly reduced credibility in medium-familiarity publishers, whether compared with no ads or non-clickbait ads. While the impact of clickbait and political ads are not significant for low familiarity publishers, the point estimates are also negative. Also, the difference between low and medium familiarity is not statistically significant. A linear hypothesis test of "low effect $=$ medium effect", using the $\chi^{2}$ test-statistic, results in $p=0.17$. Hence, the effects for low and medium-familiarity publishers are not statistically distinguishable, although there is a significant effect for medium-familiarity publishers (Gelman and Stern 2006). For high familiarity publishers the estimated effect is not statistically significant. ${ }^{12}$

As with the aggregated effects, these effects are substantial compared to other measures of heterogeneity in perceived credibility. The effect of clickbait ads for the medium familiarity publishers is four-fifths of the difference between $C N N$ and Fox News ratings, two-thirds of the maximum difference between mean ratings among all publishers, and a fourth of the difference between Republicans and Democrats' mean ratings.

\footnotetext{
${ }^{12}$ Notice that the aggregated familiarity score for Vox in Experiment 2 was $31 \%$ and differs from the one obtained from the independent survey (below 25\%). However, our results are robust to the re-classification of Vox, and become even more significant if we classify Vox per the familiarity rating of the participants of the experiments instead of the participants of the pre-survey. See Appendix 3.4.6 for more details.
} 
Figure 5 visually summarizes the above-mentioned results. Figures on the right show the proportion of clickbait and political ads in various publishers bucketed per familiarity level, as found in the descriptive analysis. Figures on the left show the estimated impact of a one-time exposure to clickbait and political ads for the same set of publishers. ${ }^{13}$ Taken together, these results signify the prevalence of these types of ads on publishers website, and potential negative effect they can have on their audience perception of credibility.

3.2.7. Readers' individual familiarity If the individual-level familiarity ratings perfectly captured the respondent's true familiarity with the source, it would be preferable to use the individual-level score. However, we know that survey measures that require reliance on memory can be noisy: "The greater he demands a question places on memory, the less accurate the respondents' answers and, all else being equal, the less accurate the survey estimates derived from them" (Tourangeau et al. 2000, p. 62). This is especially true for media use: individuals have great difficulty in accurately recalling their own past media consumption (Prior 2007, 2013). Aggregate measures of familiarity, however, allow for relative comparisons in the population across different outlets, as noisy individual survey responses can be combined to form a more reliable signal. In our analyses, we therefore relied on these aggregate measures for analysis. However, to follow our pre-registration plan, we include the results a three-way interaction model (where interaction with individual familiarity is added to model 2) in the appendix. The results show some heterogeneity based on individual familiarity with publishers, but our sample appears to be under-powered to detect significant effects for this model. For further details see Appendix 3.4.7.

3.2.8. Publishers' heterogeneity Finally, we make some observations about how the native ads impact may differ by publisher. By comparing predicted random effect and the corresponding treatment effect for each publisher, we found evidence that clickbait ads can make the credibility of best-rated publishers appear like worst-rated publishers. For example, with no ads Vox is rated significantly better than Fox News (mean difference $=0.043$ with $95 \% \mathrm{CI}=[0.009,0.0771], p=$ 0.01). However, when hosting clickbait ads, these publishers' ratings become indistinguishable (mean difference $=0.008$ with $95 \% \mathrm{CI}=[-0.093,0.109], p=0.8)$. Further, clickbait ads may significantly lower the credibility of publishers that are indistinguishable without ads. See Appendix 3.4.8 for more details.

\section{Discussion}

Native ads about politics or using a clickbait style are prevalent across many news publishers. We present evidence that these formats have an impact on perceived news credibility. Two large

\footnotetext{
${ }^{13}$ Note that publishers later used as low familiarity in the experiment were not included in the descriptive analysis.
} 
experiments provide evidence that these ubiquitous native ads have effects that conflict with the interests of publishers. The pre-experiment focused on the effects of the presence of mostly nonclickbait ads and of political ads. It provided evidence that the presence of ads impacts news credibility and that this impact varied for different stimuli. The design of Experiment 2 allowed us distinguish article and publisher effects while estimating differences between clickbait and nonclickbait ads and between political and non-political ads. This experiment showed that clickbait and political ads reduced credibility, while non-clickbait and non-political ads were no different than content presented without ads.

Perhaps unsurprisingly, widely-known publishers were not detectably impacted by a one-time exposure to native ads - suggesting that publications on which readers have the strongest prior are the least impacted by disturbing native ads, consistent with a Bayesian mechanism. Moreover, results for low familiarity publishers are more volatile: an unknown publication may sometimes benefit from embedding ads that are also found on high-familiarity / higher-credibility publications. On the contrary, medium familiarity publishers' credibility seem potentially vulnerable to a one-time exposure to ads. This argument would be consistent with curvilinear patterns of media effects where it's the medium favorable/credible/familiar outlets where you see the largest effects of changes at the margins for specific properties of the media (Iyengar and Kinder 2010, Robinson and Lance Holbert 2018). The actual mechanism that underlies how credibility perception is formed and varies for different publishers is beyond the scope of this paper, but constitutes an interesting research question for future work. Similarly, the potential effect of repetitive exposures to clickbait and political ads has not been addressed here.

Online journalism generates revenue in large part through display and native ads. In particular, native ads make up $60 \%$ of the digital-revenue stream for the publishing industry (Sirrah 2019) and this number has been increasing over the years (Laursen 2017). These native ad networks continue to sell ad spaces to many heterogeneous bidders placing political and clickbait ads. As a result, news publishers that heavily rely on native ads might trade short-term financial survival for audience loyalty. Thus, online publishers, the public, and policy-makers perhaps ought to pay close attention to the ads that appear alongside online news.

Finally, note that the question of detrimental native ads goes beyond the survival of ad-funded publications. Presence of low-quality ad-funded competitors can influence the optimal business strategy for high-quality publishers (Casadesus-Masanell and Zhu 2010). As a result, many popular newspapers, such as New York Times or Wall Street Journal, have adopted subscription-based business models and are increasingly relying on and optimizing their acquisition (e.g., Aral and Dhillon 2020) and retention (e.g., Yang et al. 2020) of subscribers - a source of revenue without, e.g., the risks of native ads. On the other hand, Chiou and Tucker (2013) show that digital paywalls can 
substantially suppress demand, and therefore might not be the optimal strategy for all publishers. If the gap between publishers that can rely on paid subscription for revenue (e.g., widely-known publishers and specialized publishers) and those that cannot increases, in can increase differences in the credibility of news available to different types of readers, with broader consequences for public education and opinion. Our results are consistent with industry ambivalence about these formats; The Atlantic, for instance, which also has paying subscribers, stopped using native ad formats in 2019. As this process continues, we hypothesize that the negative signal provided by the presence of political and clickbait native ads may only intensify.

\section{Data and replication materials}

The anonymized code and data are available at https://osf.io/48w6s/?view_only= bf5b9fd54aba4c2c8cbace77ba2037e7. This OSF repository contains 3 folders: (1) Pre-registration - contains the pre-registration plan. (2) Descriptive Analysis - contains the code and data used to scrape and analyze the native ads under study in the paper (see the README for complete details). (3) Behavioral Experiments - contains the stimuli created for both behavioural experiments, the data collected from the latter and the code used to analyse it (see the README for complete details).

\section{Acknowledgments}

We thank Yi Wang, Tesalia Rizzo, Zachary Markovic, Jesse Clark, Chris Peng and Mariette Meunier for excellent research assistance. This research benefited from early conversations with Andrey Fradkin and Markus Mobius, and from feedback from Maurice Jakesch and Zivvy Epstein.

\section{References}

Abhishek V, Jerath K, Sharma S (2019) Advertising on online marketplaces: Information asymmetry and the relevance of sponsored listings. Available at SSRN 3013468.

Amazeen MA, Wojdynski BW (2019) Reducing native advertising deception: Revisiting the antecedents and consequences of persuasion knowledge in digital news contexts. Mass Communication and Society $22(2): 222-247$.

Angelucci C, Cagé J (2019) Newspapers in times of low advertising revenues. American Economic Journal: Microeconomics 11(3):319-64.

Aral S, Dhillon PS (2020) Digital paywall design: Implications for content demand and subscriptions. Management Science.

Aribarg A, Schwartz EM (2020) Native advertising in online news: Trade-offs among clicks, brand recognition, and website trustworthiness. Journal of Marketing Research 57(1):20-34.

Athey S, Calvano E, Gans J (2013) The impact of the internet on advertising markets for news media. Technical report, National Bureau of Economic Research. 
Athey S, Gans JS (2010) The impact of targeting technology on advertising markets and media competition. American Economic Review 100(2):608-13.

Austin S, Newman N (2015) Attitudes to sponsored and branded content (native advertising). Digital News Report.

Bakshi AC (2014) Why and how to regulate native advertising in online news publications. U. Balt. J. Media L. E Ethics 4:4.

Banerjee A, Urminsky O (2022) What you are getting and what you will be getting: Testing whether verb tense affects intertemporal choices. Journal of Experimental Psychology: General.

Bashir MA, Arshad S, Wilson C (2016) "Recommended For You": A first look at content recommendation networks. Proceedings of the 2016 Internet Measurement Conference, 17-24.

Bazaco A (2019) Clickbait as a strategy of viral journalism: Conceptualisation and methods.

Beattie G, Durante R, Knight B, Sen A (2021) Advertising spending and media bias: Evidence from news coverage of car safety recalls. Management Science 67(2):698-719.

Becker-Olsen KL (2003) And now, a word from our sponsor-a look at the effects of sponsored content and banner advertising. Journal of Advertising 32(2):17-32.

Benson R (2019) Paywalls and public knowledge: How can journalism provide quality news for everyone? Journalism 20(1):146-149.

Blei DM, Ng AY, Jordan MI (2003) Latent dirichlet allocation. the Journal of machine Learning research $3: 993-1022$.

Brenan M (2016) Americans' trust in mass media edges down to 41\%. Gallup.

Campbell C, Marks LJ (2015) Good native advertising isn’t a secret. Business Horizons 58(6):599-606.

Carlson M (2015) When news sites go native: Redefining the advertising-editorial divide in response to native advertising. Journalism 16(7):849-865.

Casadesus-Masanell R, Zhu F (2010) Strategies to fight ad-sponsored rivals. Management Science 56(9):14841499.

Castillo C, Mendoza M, Poblete B (2011) Information credibility on Twitter. Proceedings of the 20th international conference on World wide web, 675-684.

Cawley A (2019) Digital transitions: The evolving corporate frameworks of legacy newspaper publishers. Journalism Studies 20(7):1028-1049.

Chatterjee P, Zhou B (2021) Sponsored content advertising in a two-sided market. Management Science.

Chiou L, Tucker C (2013) Paywalls and the demand for news. Information Economics and Policy 25(2):61-69.

Choi H, Mela CF, Balseiro SR, Leary A (2020) Online display advertising markets: A literature review and future directions. Information Systems Research 31(2):556-575. 
Cramer H (2015) Effects of ad quality \& content-relevance on perceived content quality. Proceedings of the 33rd Annual ACM Conference on Human Factors in Computing Systems, 2231-2234.

Ellman M, Germano F (2009) What do the papers sell? A model of advertising and media bias. The Economic Journal 119(537):680-704.

Epstein Z, Pennycook G, Rand D (2020) Will the crowd game the algorithm? Using layperson judgments to combat misinformation on social media by downranking distrusted sources. Proceedings of the 2020 CHI Conference on Human Factors in Computing Systems, 1-11.

Federal Trade Commission (FTC) (2015) Native advertising: A guide for businesses. URL https: //law.yale.edu/sites/default/files/area/center/isp/documents/native_advertising_a_ guide_for_businesses_federal_trade_commission.pdf.

Ferrer Conill R (2016) Camouflaging church as state: An exploratory study of journalism's native advertising. Journalism Studies 17(7):904-914.

Fink K (2019) The biggest challenge facing journalism: A lack of trust. Journalism 20(1):40-43.

Firebaugh G, Warner C, Massoglia M (2013) Fixed effects, random effects, and hybrid models for causal analysis. Handbook of causal analysis for social research, 113-132 (Springer).

Flanagin AJ, Metzger MJ (2000) Perceptions of internet information credibility. Journalism 83 Mass Communication Quarterly 77(3):515-540.

Gelman A, Stern H (2006) The difference between "significant" and "not significant" is not itself statistically significant. The American Statistician 60(4):328-331.

Gentzkow M, Shapiro JM, Sinkinson M (2011) The effect of newspaper entry and exit on electoral politics. American Economic Review 101(7):2980-3018.

Gilley S (2013) Blurred lines: Advertising or content? - an FTC workshop on native advertising. URL https://www.ftc.gov/news-events/events-calendar/2013/12/ blurred-lines-advertising-or-content-ftc-workshop-native.

Greene WH (2003) Econometric analysis (Pearson Education India).

Griffith E (2014) How Taboola and Outbrain are battling a bad reputation... and each other.

Gwet KL (2014) $t$ (Advanced Analytics, LLC).

Howe P, Teufel B (2014) Native advertising and digital natives: The effects of age and advertisement format on news website credibility judgments. ISOJ Journal 4(1):78-90.

Hurley Hall S (2019) Native ads vs. display ads. Taboola Blog, URL https://blog.taboola.com/ native-ads-vs-display-ads/.

Iversen MH, Knudsen E (2019) When politicians go native: The consequences of political native advertising for citizens' trust in news. Journalism 20(7):961-978. 
Iyengar S, Kinder DR (2010) News that matters: Television and American opinion (University of Chicago Press).

Kalveks T (2007) Clickbait. The Blackwell Encyclopedia of Sociology.

Kirchhoff SM (2010) US newspaper industry in transition (DIANE Publishing).

Knight Foundation (2018) Indicators of news media trust.

Krouwer S, Poels K, Paulussen S (2017) To disguise or to disclose? The influence of disclosure recognition and brand presence on readers' responses toward native advertisements in online news media. Journal of Interactive Advertising 17(2):124-137.

Ladd JM (2012) Why Americans Hate the Media and How It Matters (Princeton University Press).

Landis JR, Koch GG (1977) The measurement of observer agreement for categorical data. biometrics.

Laursen J (2017) Native advertising trends in news media.

Lewis RA, Reiley DH (2014) Online ads and offline sales: measuring the effect of retail advertising via a controlled experiment on yahoo! Quantitative Marketing and Economics 12(3):235-266.

Lewis SC (2020) Lack of trust in the news media, institutional weakness, and relational journalism as a potential way forward. Journalism 21(3):345-348.

Liu Z (2005) Reading behavior in the digital environment. Journal of Documentation.

Maheshwari S, Herrman J (2016) Publishers are rethinking those 'around the web' ads. The New York Times.

Marshall J (2013) Content marketing's got a quality problem.

Meyer P (1988) Defining and measuring credibility of newspapers: Developing an index. Journalism Quarterly $65(3): 567-574$.

Milgrom P (2008) What the seller won't tell you: Persuasion and disclosure in markets. Journal of Economic Perspectives 22(2):115-131.

Molina MD, Sundar SS, Le T, Lee D (2021) "fake news" is not simply false information: A concept explication and taxonomy of online content. American behavioral scientist 65(2):180-212.

Munger K (2020) All the news that's fit to click: The economics of clickbait media. Political Communication $37(3): 376-397$.

Munger K, Luca M, Nagler J, Tucker J (2020) The (null) effects of clickbait headlines on polarization, trust, and learning. Public Opinion Quarterly.

Park H, Kim S, Lee J (2020) Native advertising in mobile applications: Thinking styles and congruency as moderators. Journal of Marketing Communications 26(6):575-595.

Pennycook G, Rand DG (2019) Fighting misinformation on social media using crowdsourced judgments of news source quality. Proceedings of the National Academy of Sciences 116(7):2521-2526.

Perrin N (2019) U.S. native advertising 2019 display budgets keep flowing to new formats. 
Pew Research Center (2012) Further decline in credibility ratings for most news organizations.

Pew Research Center (2019a) Digital news fact sheet.

Pew Research Center (2019b) Newspapers fact sheet.

Prior M (2007) Post-broadcast democracy: How media choice increases inequality in political involvement and polarizes elections (Cambridge University Press).

Prior M (2013) Media and political polarization. Annual Review of Political Science 16:101-127.

Rey J (2013) Outbrain expects $25 \%$ revenue hit as it cuts on spammy content marketers.

Robinson NW, Lance Holbert R (2018) Taking sides in the war on news: exploring curvilinear associations and group differences related to perceptions of news media as threat. Journal of Applied Communication Research 46(6):684-702.

Rodgers S, Thorson E (2000) The interactive advertising model: How users perceive and process online ads. Journal of interactive advertising 1(1):41-60.

Rony MMU, Hassan N, Yousuf M (2017) Diving deep into clickbaits: Who use them to what extents in which topics with what effects? Proceedings of the 2017 IEEE/ACM international conference on advances in social networks analysis and mining 2017, 232-239.

Sahni NS (2015) Effect of temporal spacing between advertising exposures: Evidence from online field experiments. Quantitative Marketing and Economics 13(3):203-247.

Sahni NS, Nair HS (2020) Sponsorship disclosure and consumer deception: Experimental evidence from native advertising in mobile search. Marketing Science 39(1):5-32.

Sahni NS, Stanford G, Zhang C (2019) Are consumers averse to sponsored messages? The role of search advertising in information discovery. Technical report, Stanford University Graduate School of Business.

Schauster EE, Ferrucci P, Neill MS (2016) Native advertising is the new journalism: How deception affects social responsibility. American Behavioral Scientist 60(12):1408-1424.

Seamans R, Zhu F (2014) Responses to entry in multi-sided markets: The impact of Craigslist on local newspapers. Management Science 60(2):476-493.

Shariff SM, Zhang X, Sanderson M (2017) On the credibility perception of news on Twitter: Readers, topics and features. Computers in Human Behavior 75:785-796.

Shinkhede C (2019) Digital frailty: Proliferation of clickbait, beguiled readers, and questioning the morality of online journalism. marketing 6 .

Sirrah A (2019) Native advertising may jeopardize the legitimacy of newsrooms. Columbia Journalism Review.

Sisodia DS (2019) Ensemble learning approach for clickbait detection using article headline features. Informing Sci. Int. J. an Emerg. Transdiscipl. 22:31-44. 
Snyder JM, Strömberg D (2010) Press coverage and political accountability. Journal of Political Economy 118(2):355-408.

Strömbäck J, Tsfati Y, Boomgaarden H, Damstra A, Lindgren E, Vliegenthart R, Lindholm T (2020) News media trust and its impact on media use: Toward a framework for future research. Annals of the International Communication Association 44(2):139-156.

Sun M, Zhu F (2013) Ad revenue and content commercialization: Evidence from blogs. Management Science 59(10):2314-2331.

Svetlik J (2016) Impacts and effectiveness of negative political advertising in czech environment. Political Preferences.

Tourangeau R, Rips LJ, Rasinski K (2000) The psychology of survey response.

Tseng S, Fogg B (1999) Credibility and computing technology. Communications of the ACM 42(5):39-44.

Usher N (2018) Re-thinking trust in the news: A material approach through "objects of journalism". Journalism Studies 19(4):564-578.

Wang P, Xiong G, Yang J (2019) Serial position effects on native advertising effectiveness: Differential results across publisher and advertiser metrics. Journal of Marketing 83(2):82-97.

Wojdynski B (2019) Native advertising. Oxford Research Encyclopedia of Communication.

Wolf M (2018) Skim reading is the new normal. The effect on society is profound. The Guardian.

Wölker A, Powell TE (2021) Algorithms in the newsroom? News readers' perceived credibility and selection of automated journalism. Journalism, URL http://dx.doi.org/10.1177/1464884918757072.

Wu M, Huang Y, Li R, Bortree DS, Yang F, Xiao A, Wang R (2016) A tale of two sources in native advertising: Examining the effects of source credibility and priming on content, organizations, and media evaluations. American Behavioral Scientist 60(12):1492-1509.

Yale RN, Jensen JD, Carcioppolo N, Sun Y, Liu M (2015) Examining first-and second-order factor structures for news credibility. Communication Methods and Measures 9(3):152-169.

Yang J, Eckles D, Dhillon P, Aral S (2020) Targeting for long-term outcomes. arXiv preprint arXiv:2010.15835.

Zhou Y (2017) Clickbait detection in tweets using self-attentive network. arXiv preprint arXiv:1710.05364. 